\title{
Psicooncología
}

ISSN: 1696-7240

\section{Intervención cognitivo-conductual en cuidados paliativos pediátricos: un caso clínico}

\author{
Alberto Gómez Zarco; Ana Leticia Becerra Gálvez²; Rocio Tron Álvarez³ Patricia \\ Hernández Solís ${ }^{4, *}$
}

Recibido: 13 de febrero de 2018 / Aceptado: 20 de agosto de 2018

Resumen: Objetivo: El cáncer infantil es una enfermedad que modifica el estilo y calidad de vida de los niños y sus familias debido al impacto físico, emocional y económico que provoca. Los Cuidados Paliativos Pediátricos tienen entre sus funciones otorgar atención psicológica a los niños y sus familiares desde la etapa prediagnóstica con la finalidad de mejorar el estado anímico del paciente y promover pensamientos y conductas que le permitan adaptarse a su nueva condición y mejoren la adherencia al tratamiento. Se expone el caso de una paciente pediátrica con diagnóstico de Sarcoma Sinovial que presenta sintomatología depresiva y evitación ansiosa a raíz de su padecimiento. Método: La evaluación se realizó mediante entrevista conductual y observación directa de la sintomatología ansiosa y depresiva intrahospitalaria y la Escala Visual Análoga (EVA) para el dolor. El tratamiento se llevó a cabo en siete sesiones en las que se emplearon técnicas de la Terapia Cognitivo-Conductual. Resultados: Entre los resultados se obtuvieron verbalizaciones adaptativas sobre su padecimiento, posibles tratamientos médicos, autoconcepto, una mejora en la expresión emocional y control del dolor. Conclusiones: El presente caso es una muestra de la importancia que tiene la labor del psicólogo en los Cuidados Paliativos Pediátricos, así comoel impacto del trabajo con los niños que padecen enfermedades oncológicas y sus familiares. La Terapia CognitivoConductual ha demostrado ser altamente eficaz en este tipo de pacientes, aún en intervenciones breves. Palabras Clave: Cuidados paliativos pediátricos; terapia cognitivo-conductual; cáncer infantil; ansiedad; depresión y estrés.

\section{[en] Cognitive-behavioral intervention in pediatric palliative care: a clinical case}

Abstract: Objective: Childhood cancer is a disease that modifies the style and quality of life of children and their families due to the physical, emotional and financial impact it has on them. Pediatric Palliative Care has among its functions the granting of psychological attention to children and their families from

1 Alberto Gómez Zarco. Facultad de Estudios Superiores Iztacala. Universidad Nacional Autónoma de México. Calle Lago Leticia. Manzana 23 F. Lote 10. Colonia Ampliación Ciudad Lago. C.P. 57185. Nezahualcóyotl. Estado de México. México.

E-mail: psic.albertogomez@gmail.com

2 Ana Leticia Becerra Gálvez. Facultad de Estudios Superiores Iztacala. Universidad Nacional Autónoma de México. México.

E-mail: behaviormed.ana@gmail.com

3 Rocio Tron Álvarez. Facultad de Estudios Superiores Iztacala. Universidad Nacional Autónoma de México. México. E-mail: rociotron@hotmail.com

4 Patricia Hernández Solís. Clínica del Dolor y Cuidados Paliativos. Hospital Juárez de México. México. E-mail: aaaph2000@prodigy.net.mx

* Dirección de correspondencia: Patricia Hernández Solís. Clínica del Dolor y Cuidados Paliativos. Hospital Juárez de México. México. E-mail: aaaph2000@prodigy.net.mx 
the pre-diagnostic stage until terminal stage. We present the case of a pediatric patient diagnosed with Synovial Sarcoma who presents depression and anxious avoidance as a result of her medical condition. Method: The evaluation was carried out through a behavioral interview, direct observation of anxiety and depression responses in the hospital and Visual Analogue Scale (VAS) for pain. The treatment consisted of seven sessions of intervention with Cognitive-Behavioral Therapy techniques. Results: The obtained results include adaptive verbalizations about their condition, possible medical treatments, self-concept, an improvement in emotional expression and management of pain. Conclusion: This case is a sample of the importance of the work of the psychologist in Pediatric Palliative Care, as well as the impact of working with children suffering from oncological diseases and their families. Cognitive-Behavioral Therapy has been shown to be highly effective in this type of patients, even in brief interventions.

Keywords: Pediatric palliative care; cognitive-behavioral therapy; pain; anxiety and depression.

Sumario. 1. Introducción 2. Presentación del Caso Clínico 2.1. Datos generales de identificación 2.2. Dinámica Familiar. 2.3. Historia Médica y Psicológica. 3. Formulación del Caso Clínico 3.1. Motivo de Consulta. 3.2. Evaluación Clínica Psicológica. 3.3. Análisis de Variables y Secuencias Funcionales relacionadas al Problema. 4. Plan de Intervención 5. Resultados 6. Conclusiones y Discusión 7. Referencias bibliográficas

Cómo citar: Gómez Zarco A, Becerra Gálvez AL, Tron Álvarez R, Hernández Solís P. Intervención cognitivo-conductual en cuidados paliativos pediátricos: un caso clínico. Psicooncología 2018;15:385398. doi: 10.5209/PSIC.61444.

\section{Introducción}

En México, el cáncer es la principal causa de muerte en pacientes entre los 5 y 14 años y cerca del $65 \%$ de los infantes son diagnosticados en etapas avanzadas de la enfermedad, por lo cual su atención integral es fundamental ${ }^{(1,2)}$. Esta enfermedad genera una serie de alteraciones físicas, psicológicas y sociales en los niños y sus familiares, entre las que se encuentran un incremento en la dependencia y pérdida de la funcionalidad, cambios en la imagen corporal y relaciones interpersonales, incapacidad para la realización de las actividades cotidianas y dolor. Dichas alteraciones vulneran su calidad de vida y hacen imprescindible la asistencia interdisciplinaria del área de Cuidados Paliativos ${ }^{(3)}$.

Los Cuidados Paliativos tienen como propósito mejorar la calidad de vida del paciente y movilizar los recursos familiares para favorecer el mejor ajuste a la enfermedad. Al respecto la Organización Mundial de la Salud (OMS) menciona que los Cuidados Paliativos deben incluir atención médica, psicológica, social y espiritual por lo que, el trabajo interdisciplinario se vuelve fundamental en esta etapa de la enfermedad. Las intervenciones con enfoque paliativo van más allá del control de los síntomas derivados de la enfermedad y el tratamiento, incluyen un enfoque activo otorgando al paciente la posibilidad de conocer la información que le permita la toma de decisiones sin forzar su elección, aun cuando sea niño o adolescente ${ }^{(4,5)}$. Este tipo de poblaciones son susceptibles a vivir una mayor prevalencia de trastornos psicológicos (principalmente por el desconocimiento de los padres en relación a la sobrevida y el objetivo del tratamiento), obstinación terapéutica y un déficit importante en la atención psicosocial. Fue por esto que la OMS enfatiza la importancia de la atención en Cuidados Paliativos Pediátricos (CPP), los cuales tienen como objetivo garantizar atención integral por parte de los profesionales de la salud en este estrato poblacional. 
A pesar de los beneficios que otorgan los CPP, los esfuerzos realizados por diversas instituciones y la implementación de diversas políticas de salud en México, las clínicas de CPP son escasas y las que existen no poseen la infraestructura e insumos necesarios, por lo que el acceso a este servicio es limitado ${ }^{(4,6)}$. Ante este fenómeno, es responsabilidad del personal de salud disponible proporcionar atención de calidad desde el diagnóstico hasta el final de la vida, dado que, de no hacerlo, esto representará un enorme coste físico y emocional en los pacientes pediátricos y sus familiares, aumentando el riesgo de ansiedad, depresión, angustia, miedo y sufrimiento $^{(4,5,7)}$.

El psicólogo que labora en CPP debe tener en cuenta que su intervención es totalmente diferente a la que se realiza con un adulto, por lo que debe formular objetivos claros y utilizar técnicas de intervención eficaces y basadas en evidencia. Para cumplir con dichas actividades el psicólogo deberá considerar las siguientes variables:

Edady desarrollo cognitivo- emocional del infante. La estructura emocional de un menor se encuentra en formación, lo cual que tiene un impacto importante en la visión que tiene de la enfermedad, la comprensión de todo lo que sucede a su alrededor, la expresión emocional y de los síntomas relacionados al padecimiento ${ }^{(5,7-10)}$.

Emociones y cogniciones derivadas del padecimiento. Es importante considerar los deseos, valores, creencias, capacidad de razonamiento y toma de decisiones del menor durante el tratamiento. La intervención psicológica independientemente del enfoque teórico que la sustente debe estar basada en los preceptos básicos de respeto, delicadeza, honestidad y comprensión del menor con la finalidad de lograr una primera y fuerte alianza terapéutica con el infante ${ }^{(5,7-10)}$.

Variables relacionadas a la enfermedad y tratamiento. El cáncer infantil cambia drásticamente el estilo de vida del paciente y su familia puesto que desde el pre-diagnóstico y durante todo el tratamiento el niño se enfrenta a una serie de condiciones que le generan dolor, estrés y malestar. Los procedimientos médicos y los frecuentes ingresos hospitalarios son estresores que pueden durar desde meses hasta años mermando la funcionalidad, imagen corporal, autoconcepto y calidad de vida del niño ${ }^{(8,9,11)}$.

Condiciones ambientales e interacción familiar. Conocer la dinámica familiar del menor y las diferentes redes de apoyo resulta indispensable, puesto que permite identificar la información de la que disponen y evitar la denominada conspiración del silencio. Los padres del niño pueden creer que ocultar información lo protege de sufrir angustia o depresión, sin embargo, en muchas ocasiones esta acción se traduce en enojo, distanciamiento emocional, poca comprensión de la enfermedad, creencias erróneas respecto al pronóstico y dificultad para que el infante resuelva sus dudas ${ }^{(7,8)}$.

Cada uno de estos elementos deberá constituir parte del Análisis Funcional de la Conducta del paciente y así direccionar las estrategias de intervención a utilizar. En el caso de la etapa post-diagnóstica (aun cuando se trata de un paciente paliativo), se debe proporcionar psicoeducación sobre la enfermedad, el tratamiento y sus posibles efectos secundarios, favorecer la expresión emocional y establecer canales de comunicación entre el paciente y los familiares, optimizar el apego al tratamiento, disminuir el malestar psicológico y evitar la toma de decisiones precipitadas que pueden afectar el tratamiento médico ${ }^{(5,8,11-14)}$.

Como hemos visto hasta el momento, el trabajo psicológico en CPP debe ser llevado a cabo desde el momento previo al diagnóstico puesto que en esta etapa el 
paciente se encuentra más vulnerable debido al impacto psicológico que produce la enfermedad, y debe tener como finalidad el reducir la sintomatología ansiosodepresiva y el estrés al que se enfrentan constantemente. Uno de los modelos que ha probado eficacia y del cual se dispone evidencia empírica de su utilidad en este tipo de variables es la Terapia Cognitivo-Conductual, la cual parte de que los problemas de adaptacióm en los pacientes de derivan de sus pensamientos, emociones y comportamientos. Es por lo anterior que el objetivo del presente estudio es describir los resultados de una intervención psicológica basada en componentes de la Terapia Cognitivo-Conductual en una paciente pediátrica paliativa con Sarcoma Sinovial la cual fue atendida en la Clínica del Dolor y Cuidados Paliativos del Hospital Juárez de México.

\section{Presentación del Caso Clínico}

\subsection{Datos generales de identificación}

Paciente femenino de 10 años de edad a quién para fines de confidencialidad llamaremos Selene, la cual cuenta con diagnóstico de Sarcoma Sinovial de Miembro Torácico Izquierdo, estudiante de $6^{\circ}$ año de educación primaria, originaria de la Ciudad de México, residente del Estado de México y de religión católica.

\subsection{Dinámica Familiar}

Durante la entrevista con familiares, se encuentra que la paciente vive con su madre y dos hermanas, así como con su abuela y tío materno, con quienes tiene buena comunicación. Los padres de Selene son divorciados por lo que la relación con su padre suele ser distante y poco funcional según advirtió la menor (Figura 1).

Figura 1. Genograma de la paciente

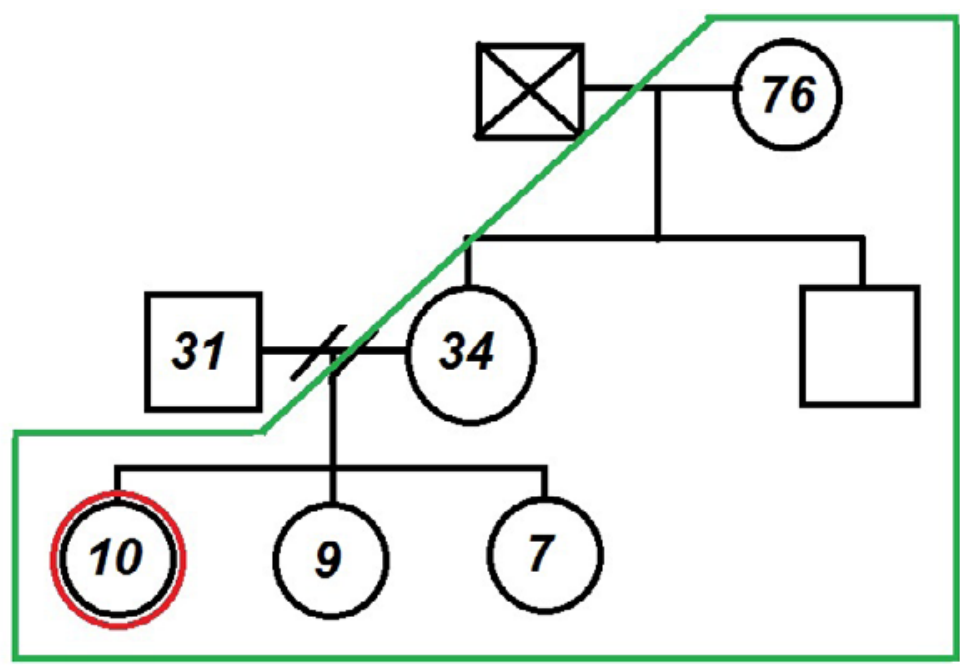




\subsection{Historia Médica y Psicológica}

El padecimiento médico inició con una contusión directa en estructura metálica sobre el Miembro Torácico Izquierdo (MTI), el cual presenta aumento de volumen progresivo. Después de la valoración del médico general, se descartó fractura, sin embargo, ante la persistencia de dolor y malestar general fue trasladada a un hospital de segundo nivel de atención, donde identificaron un número elevado de lesiones blásticas lo que sugería la presencia de un probable osteosarcoma de tipo medular metastásico.

Por último, Selene fue atendida por los servicios de Ortopedia y Oncología Pediátrica del Hospital Juárez de México y el servicio de Clínica del Dolor y Cuidados Paliativos fue interconsultado para valoración y control del dolor por toma de biopsia, ya que la paciente refiere una Escala Visual Análoga del Dolor (EVA) $8 / 10$. Se proporcionó tratamiento para el dolor con infusión de Buprenorfina $200 \mathrm{mg}$ + Metamizol 1,5 mg + Lidocaína $40 \mathrm{mg}$ en $100 \mathrm{ml}$ de solución de $\mathrm{NaCl}$ (Cloruro de Sodio) al 9\% para 24 horas y Oxcarbacepina en suspensión $5 \mathrm{ml}$ cada 12 horas. Debido a continuidad del dolor (EVA 3/10), se agregó Imipramina tabletas de $25 \mathrm{mg} \mathrm{1/4}$ por la noche y se modificó la dosis de Oxcarbacepina a $5 \mathrm{ml}$ cada 8 horas.

Una vez confirmado el Sarcoma Sinovial en MTI por resultado histopatológico, el servicio de Oncología pediátrica suguirió como estrategia de tratamiento la desarticulación previa a la toma de quimioterapia, mientras que Ortopedia pediátrica consideraba que la quimioterapia previa permitiría la disminución del tamaño de la tumoración y esto mejoraría la calidad de la cirugía. Pese a ambas propuestas terapéuticas los padres decidieron trasladar a la paciente al estado de San Luis Potosí (por recomendación de sus familiares), por lo que en el Hospital Juárez de México no fue realizado algún tratamiento médico curativo para su padecimiento por los servicios de oncología pediátrica y ortopedia.

La paciente niega antecedentes psicológicos de importancia, así como atención psicológica-psiquiátrica previa a la que recibió por parte del psicólogo de la Clínica de Dolor y Cuidados Paliativos del Hospital Juárez de México.

\section{Formulación del Caso Clínico}

\subsection{Motivo de Consulta}

La paciente fue entrevistada durante el pase de visita realizado por psicólogo de la Clínica de Dolor y Cuidados Paliativos a sugerencia de personal médico y ante probable diagnóstico médico y terapéutica. Al tratarse de una menor de edad y considerando su probable enfermedad se proporcionó seguimiento a paciente y familiares.

\subsection{Evaluación Clínica Psicológica}

Laevaluaciónpsicológicase realizómediante entrevista conductual semiestructurada realizada con la paciente y ambos padres, así como observación clínica directa en 
el contexto hospitalario, lugar donde se llevó a cabo la intervención. La evolución del dolor se llevó a cabo con el uso de la Escala Visual Análoga del Dolor (EVA).

\subsection{Análisis de Variables y Secuencias Funcionales relacionadas al Problema}

Para la identificación de las secuencias funcionales relacionadas al problema, se utilizó el Mapa de Patogénesis propuesto por Nezu, Nezu y Lombardo ${ }^{(15)}$ el cual consiste en una descripción gráfica de las variables hipotéticas relacionadas al paciente, al ambiente, al tiempo y a la interacción funcional entre cada una de ellas. Esta herramienta permite al clínico identificar las causas que contribuyen al surgimiento y mantenimiento de las dificultades del paciente generando así alternativas para la solución de sus problemas. En la Figura 2 podemos observar una síntesis de la relación existente entre las diversas variables, respuestas y consecuencias analizadas en el caso de Selene.

Al momento de la evaluación, la paciente presentaba como variables de respuesta facie de tristeza, afecto hipotímico, sentimientos de tristeza, respuestas motoras de depresión como llanto contenido, evitación para hablar del diagnóstico médico, poco contacto visual y verbalizaciones como "no quiero hablar de eso porque me pongo triste”, "me gustaría estar con mis hermanas y jugar con ellas... quiero decirles que las quiero", "quiero ver a mis hermanas y mi abuelita porque también está enferma”, así como respuestas motoras de ansiedad y estrés, tales como respiración agitada, gritos y movimientos para evitar procedimientos y verbalizaciones relacionadas a estas emociones como "estoy aburrida, no tengo nada que hacer", "ya me quiero ir", "no quiero saber qué tengo porque me pondría más nerviosa", "los doctores hablaron con mis papás y vi a mi mamá muy triste... creo que es algo malo" y "no quiero que me lastimen más"

Como podemos observar en la Figura 2, estas respuestas tienen como variables distales el traumatismo directo con estructura metálica en el MTI (mayo de 2016), presencia de dolor constante que no remite y las múltiples valoraciones y procedimientos médicos en diferentes instituciones a los que Selene se vio expuesta previo al diagnóstico. Las respuestas de Selene se ven mediadas por la presencia de dolor incidental en MTI, el diagnóstico de Sarcoma Sinovial, un estilo de afrontamiento evitativo y sus creencias sobre la génesis de la enfermedad (por traumatismo). Otros factores influyentes en la respuesta adaptativa fueron la relación cercana con familiares (redes de apoyo adecuadas y aparentemente funcionales) y su papel como cuidadora de su abuela amputada de miembro pélvico izquierdo (vivir la experiencia de su abuela permitió a Selene identificarse y visualizar su posible amputación).

Las respuestas de depresión, estrés y ansiedad se presentaban al pensar o escuchar el probable diagnóstico, recordar o escuchar sobre su familia, los días de estancia intrahospitalaria y procedimientos médicos invasivos (colocación de catéter largo IV), siendo mantenidas debido a que los familiares y personal médico no hablaban detalladamente sobre el diagnóstico o tratamiento y emitían palabras de consuelo ante las verbalizaciones de dolor (ver Figura 2).

Una vez realizada la evaluación se estableció como objetivo generar en la paciente respuestas adaptativas ante las estancia hospitalaria, diagnóstico y tratamiento médicos, 


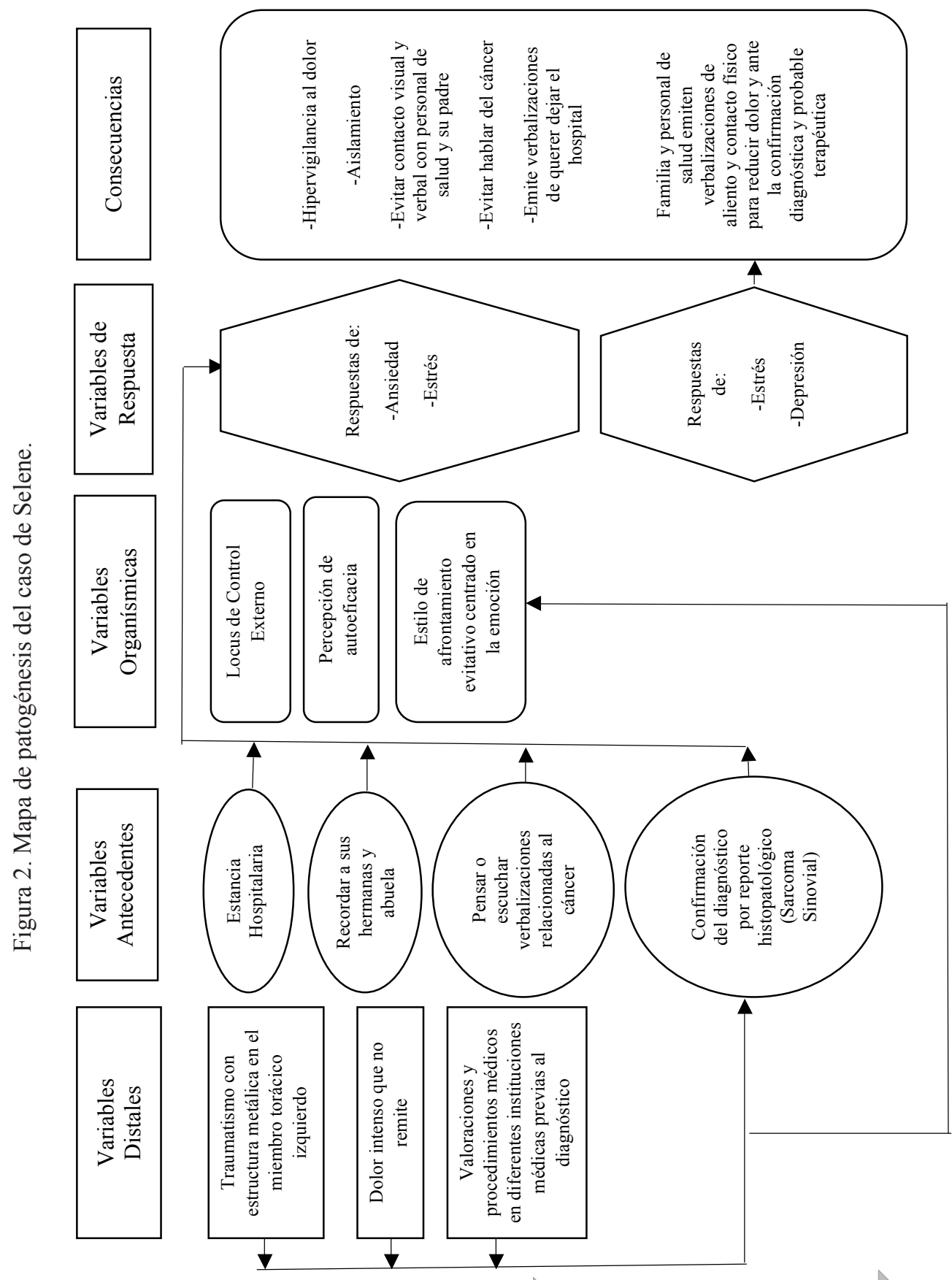




\section{Plan de Intervención}

El plan de intervención se dividió en dos fases, la primera durante la etapa prediagnóstico médico, en la cual se buscó establecer componentes de relación funcionales con la paciente para incorporar las estrategias de intervención de manera efectiva, para lograr una adecuada expresión emocional (mediante la escritura de cartas y juego de roles para expresar sus dudas, preocupaciones y deseos a sus familiares), identificar las creencias respecto al origen del padecimiento médico tanto en Selene como en sus familiares mediante la entrevista y proporcionar psicoeducación ajustada a sus necesidades y dudas. Un objetivo secundario que se persiguió en esta fase fue una reducción en la hipervigilancia al dolor y un mejor ajuste a la estancia hospitalaria prolongada mediante distracción instrumental (realización de papiroflexia y pulseras para regalar a sus familiares).

En la fase diagnóstica se identificaron las creencias relacionadas al pronóstico del cáncer y sus modalidades de tratamiento mediante la entrevista, mientras que el objetivo que se persiguió fue identificar con apoyo de la paciente las capacidades y competencias de las que podría disponer de llevarse a cabo la amputación del MTI (mediante el dibujo de sí misma después de la amputación, la verbalización de sus valores y habilidades y tomando como ejemplo a su abuela amputada de Miembro Pélvico Izquierdo). Debido a la importancia que tienen los padres en el tratamiento médico y psicológico con pacientes pediátricos, se realizó entrenamiento en habilidades de comunicación asertiva para mejorar su relación con la paciente mediante juego de roles y evitar la conspiración del silencio mediante psicoeducación, así como la identificación de ventajas y desventajas para la toma de decisiones respecto al tratamiento de Selene empleando la técnica de solución de problemas. Los detalles del plan de intervención pueden observarse en la Tabla 1.

Tabla 1. Intervención basada en técnicas Cognitivo-Conductuales por sesiones.

\begin{tabular}{|c|c|c|c|}
\hline Sesión & Objetivos de la sesión & Técnicas empleadas & Observaciones \\
\hline \multirow{4}{*}{1} & Evaluar conductas problema & $\begin{array}{l}\text { Entrevista conductual con paciente y } \\
\text { madre }\end{array}$ & \multirow[t]{4}{*}{$\begin{array}{l}\text { La paciente se muestra evitativa a } \\
\text { hablar sobre padecimiento médico. }\end{array}$} \\
\hline & $\begin{array}{l}\text { Informar a la madre sobre la labor del } \\
\text { psicólogo en el contexto hospitalario }\end{array}$ & Información & \\
\hline & $\begin{array}{l}\text { Establecer componentes de relación } \\
\text { con la paciente }\end{array}$ & $\begin{array}{l}\text { Conversación sobre temas de interés } \\
\text { para la paciente (como caricaturas, jue- } \\
\text { gos y materias escolares preferidas) }\end{array}$ & \\
\hline & $\begin{array}{l}\text { Disminuir sintomatología ansiosa en la } \\
\text { paciente ante la estancia hospitalaria y } \\
\text { episodios de dolor }\end{array}$ & $\begin{array}{l}\text { Distracción instrumental con la realiza- } \\
\text { ción de animales de papiroflexia }\end{array}$ & \\
\hline 2 & $\begin{array}{l}\text { Establecer componentes de relación } \\
\text { con la paciente }\end{array}$ & $\begin{array}{l}\text { Conversación sobre temas de interés } \\
\text { para la paciente (como caricaturas, jue- } \\
\text { gos y materias escolares preferidas) }\end{array}$ & $\begin{array}{l}\text { La paciente muestra mejoría de esta- } \\
\text { do anímico, pero mantiene evitación } \\
\text { ansiosa }\end{array}$ \\
\hline
\end{tabular}




\begin{tabular}{|c|c|c|c|}
\hline \multirow{2}{*}{2} & $\begin{array}{l}\text { Disminuir sintomatología ansiosa en } \\
\text { la paciente ante estancia hospitalaria y } \\
\text { episodios de dolor }\end{array}$ & $\begin{array}{l}\text { Distracción instrumental con la realiza- } \\
\text { ción de animales de papiroflexia }\end{array}$ & \multirow[t]{2}{*}{$\begin{array}{l}\text { El padre verbaliza posibles variables } \\
\text { que mantienen la percepción de do- } \\
\text { lor en la paciente. }\end{array}$} \\
\hline & $\begin{array}{l}\text { Psicoeducar al padre sobre componen- } \\
\text { tes físicos y psicológicos que influyen } \\
\text { en la percepción del dolor de Selene }\end{array}$ & Psicoeducación & \\
\hline \multirow{3}{*}{3} & $\begin{array}{l}\text { Mantener componentes de relación con } \\
\text { la paciente }\end{array}$ & $\begin{array}{l}\text { Conversación sobre temas de interés } \\
\text { para la paciente (como caricaturas, jue- } \\
\text { gos y materias escolares preferidas) }\end{array}$ & \multirow{3}{*}{$\begin{array}{l}\text { La paciente reporta una disminu- } \\
\text { ción en las repuestas de ansiedad e } \\
\text { interactúa con mayor fluidez con su } \\
\text { padre }\end{array}$} \\
\hline & $\begin{array}{l}\text { Disminuir sintomatología ansiosa en } \\
\text { la paciente ante estancia hospitalaria y } \\
\text { episodios de dolor }\end{array}$ & $\begin{array}{l}\text { Distracción instrumental con la realiza- } \\
\text { ción de pulseras }\end{array}$ & \\
\hline & $\begin{array}{l}\text { Establecer habilidades de comunica- } \\
\text { ción asertiva entre los padres de Selene }\end{array}$ & $\begin{array}{l}\text { Entrenamiento en habilidades de comu- } \\
\text { nicación asertiva }\end{array}$ & \\
\hline 4 & $\begin{array}{l}\text { Establecer en la paciente habilidades } \\
\text { de expresión asertiva de emociones y } \\
\text { sentimientos }\end{array}$ & $\begin{array}{l}\text { Entrenamiento en habilidades sociales } \\
\text { y asertividad }\end{array}$ & $\begin{array}{l}\text { La paciente practica formas de co- } \\
\text { municación para resolución de du- } \\
\text { das y preocupaciones con su madre } \\
\text { y personal de salud }\end{array}$ \\
\hline \multirow{4}{*}{5} & $\begin{array}{l}\text { Disminuir respuesta de estrés agudo en } \\
\text { los padres debida al diagnóstico médico } \\
\text { de Selene }\end{array}$ & $\begin{array}{l}\text { Intervención en crisis mediante la dis- } \\
\text { minución de respuestas emocionales } \\
\text { con Técnica de respiración diafragmá- } \\
\text { tica y validación de las emociones pre- } \\
\text { sentes ante el diagnóstico médico }\end{array}$ & \multirow{2}{*}{$\begin{array}{l}\text { Los padres no llegan a un acuerdo } \\
\text { sobre continuar tratamiento en la } \\
\text { institución o trasladarla a otro hos- } \\
\text { pital, por lo que se pospone toma de } \\
\text { decisiones y se trabaja en búsqueda } \\
\text { de información con personal médi- } \\
\text { co. }\end{array}$} \\
\hline & $\begin{array}{l}\text { Entrenar a los padres de Selene en es- } \\
\text { trategias para la toma de decisiones } \\
\text { informadas }\end{array}$ & $\begin{array}{l}\text { Identificación de Ventajas y desventajas } \\
\text { de los tratamientos médicos (balance } \\
\text { decisional) }\end{array}$ & \\
\hline & $\begin{array}{l}\text { Psicoeducar a la paciente sobre origen } \\
\text { de tumoraciones y tratamientos para el } \\
\text { cáncer }\end{array}$ & Psicoeducación & \multirow{2}{*}{$\begin{array}{l}\text { La paciente es capaz de verbalizar } \\
\text { probables orígenes de su tumoración } \\
\text { y consecuencias de tratamientos } \\
\text { médicos } \\
\text { La paciente verbaliza por lo menos } \\
\text { una alternativa de solución a cada } \\
\text { situación planteada }\end{array}$} \\
\hline & $\begin{array}{l}\text { Generar en la paciente estrategias de } \\
\text { afrontamiento adaptativas a través del } \\
\text { planteamiento de situaciones hipotéti- } \\
\text { cas de llevarse a cabo la desarticulación }\end{array}$ & $\begin{array}{l}\text { Solución de Problemas: generación de } \\
\text { alternativas de solución ante situaciones } \\
\text { de ocurrencia probable }\end{array}$ & \\
\hline 6 & $\begin{array}{l}\text { Disminuir síntomas de estrés agudo en } \\
\text { la paciente ante procedimiento medico } \\
\text { invasivo (Colocación de Catéter) }\end{array}$ & $\begin{array}{l}\text { Respiración diafragmática profunda } \\
\text { Distracción cognitiva (se inventa un } \\
\text { cuento entre el psicólogo y la paciente } \\
\text { sobre un conejo que enfrenta sus mie- } \\
\text { dos) }\end{array}$ & $\begin{array}{l}\text { Se pospone intervención centrada } \\
\text { en la generación de alternativas de } \\
\text { solución y afrontamiento debido a } \\
\text { respuesta de estrés agudo durante la } \\
\text { colocación de catéter largo. }\end{array}$ \\
\hline 7 & $\begin{array}{l}\text { Identificar con paciente y familiares } \\
\text { alternativas de solución ante problemas } \\
\text { cotidianos de llevarse a cabo la desar- } \\
\text { ticulación. } \\
\text { Conocer el tipo de autoconcepto y } \\
\text { probables alteraciones en la imagen } \\
\text { corporal de Selene de llevarse a cabo la } \\
\text { desarticulación. }\end{array}$ & $\begin{array}{l}\text { Psicoeducación } \\
\text { Solución de Problemas: generación de } \\
\text { alternativas de solución ante situaciones } \\
\text { de ocurrencia probable } \\
\text { Dibujo de sí misma sin el Miembro To- } \\
\text { rácico Izquierdo. }\end{array}$ & $\begin{array}{l}\text { La paciente es capaz de verbalizar } \\
\text { capacidades y competencias ante la } \\
\text { probable desarticulación. } \\
\text { Se informa al servicio de Clínica del } \\
\text { Dolor y Cuidados Paliativos tras- } \\
\text { lado de paciente a otra institución } \\
\text { dado que los padres toman la deci- } \\
\text { sión de trasladar a la paciente a otra } \\
\text { institución. }\end{array}$ \\
\hline
\end{tabular}




\section{Resultados}

Entre los resultados se logró la modificación de su evitación ansiosa para hablar tanto del padecimiento médico como de sus emociones y cogniciones relacionadas al cáncer. Posterior a las entrevistas e intervención se obtuvieron verbalizaciones adaptativas en Selene, sobre sí misma y sobre la posible amputación. Se observaron cambios clínicamente significativos en términos del dolor percibido y sentimientos de tristeza (disminución de las quejas verbales). Un indicador del cambio clínico logrado fueron las verbalizaciones de la madre, quien refirió durante la última sesión "ya pudimos hablar con ella y creo que recibió bien la noticia... si se puso un poco triste, pero no como pensábamos que sería" (Sic. Fam.).

Entre las verbalizaciones de la paciente referentes a su estancia intrahospitalaria, cambiaron de ser "no quiero hablar contigo" "ya no quiero estar aqui" (refiriéndose al hospital) a ser del tipo "me sentí mejor, ya no me aburrí" y "me gustó lo que hice ayer". En relación a sus sentimientos, emociones y preocupaciones, las verbalizaciones también tuvieron cambios ya que de referir "mi papá no me cree cuando me duele... me regaña y me dice que miento o se sale" y "me dicen que mi abuelita está bien, pero no les creo... ella está muy enferma" a mencionar "ya hablé por teléfono con mi abuelita y mis hermanas...me dijeron que están bien" y "ya les escribi cartas y les voy a escribir más para decirles que las quiero y saber cómo están", mientras que en lo que concierne a su enfermedad y tratamiento se modificaron de "no quiero hablar de eso porque me pongo triste" y "creo que es algo malo, pero no quiero saber porque me voy a poner más nerviosa" a ser del tipo "aunque me gustaría que no me quitaran el brazo, sé que si lo pueden hacer", "si me quitan mi brazo va a ser difícil... pero puedo aprender a hacer cosas sin él", "mi mamá dice que soy muy fuerte, y yo sé que soy valiente", "voy a poder hacer cosas que me gustan, como jugar, ir a la escuela y cuidar a mi abuelita" (Sic. Pac.).

En relación a la presencia del dolor, en la Figura 2 podemos observar que se mantuvo en niveles bajos y estables (entre 0 y 2 ), con un pico máximo en la sesión 6, durante la cual se realizó la colocación del catéter largo en la paciente. Es importante recordar que durante la etapa diagnóstica y especialmente durante las hospitalizaciones, los pacientes se enfrentan a una serie de procedimientos médico invasivos (inyecciones, canalizaciones, tomas de muestras, biopsias, etc.), por lo que el control de la ansiedad y el dolor resulta complicado. Otros factores presentes en la estancia intrahospitalaria son los tiempos prolongados en cama, estar lejos de casa y la familia y la ambigüedad y poca comprensión de los tecnicismos médicos en las explicaciones que proporcionan, por lo que se pueden generar ideas disfuncionales respecto a su padecimiento exacerbando la hipervigilancia a los síntomas físicos entre ellos el dolor.

Al finalizar la intervención, se pudo observar por una parte que con la paciente se logró la disminución de la sintomatología ansiosa y depresiva, de las conductas asociadas al dolor (llanto, hipervigilancia y verbalizaciones sobre el dolor), fue capaz de hablar de su diagnóstico médico, tratamiento y pronóstico, mejoró su capacidad de expresión de dudas, preocupaciones y emociones a sus familiares, así como de las capacidades físicas y psicológicas con las que contaría después de realizada la amputación, sin embargo, este último punto no se trabajó con la profundidad necesaria debido al traslado de la paciente a otro centro hospitalario. Por otro lado, con los pares se logró una mejoría en sus habilidades de comunicación asertiva (tanto entre 
ellos como con Selene), en su búsqueda de información con el personal de salud y su toma de decisiones sobre el tratamiento de la paciente, sin embargo, seria importante reforzar la comunicación con la paciente para evitar la conspiración del silencio en caso de dificultades durante el tratamiento médico, ya que aún cuando el pronóstico de la paciente era bueno para la vida a corto y mediano plazo en caso de realizarse de forma pronta la cirugía y quimioterapias, no pudo ser abordado a profundidad debido a su traslado a San Luis Potosí para continuar el tratamiento.

Figura 3. Percepción de dolor de la paciente por sesión.

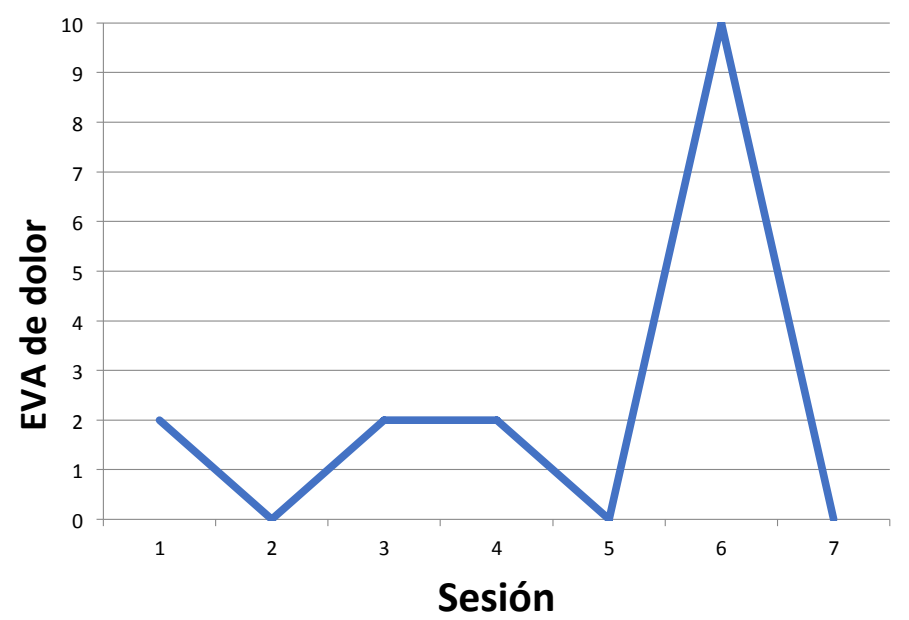

\section{Conclusiones y Discusión}

El trabajo realizado con Selene obtuvo buenos resultados durante la estancia intrahospitalaria, debido a que se logró abordar los aspectos médicos de su padecimiento y las alternativas de tratamiento. La paciente reportó un cambio en el estado anímico y una disminución en los niveles de dolor percibido. Dadas las condiciones médicas y considerando que no se contaba con la confirmación de un diagnóstico se buscó establecer componentes de relación funcionales e implementar técnicas con enfoque cognitivo-conductual para favorecer la adaptación a la estancia hospitalaria, generar un conocimiento claro respecto al probable diagnóstico y las modalidades de tratamiento y así disipar la presencia de pensamientos catastróficos o ideas equivocadas en relación al origen de la enfermedad y el pronóstico.

Becerra $^{(12)}$ y Bravo ${ }^{(11)}$ refieren que el trabajo desde la etapa prediagnóstica es de suma importancia para la mejora no sólo del estado anímico, sino también para ayudar a la adherencia al tratamiento, por lo que proponen estrategias de intervención que facilitan dicho proceso. Técnicas con enfoque cognitivo conductual como la psicoeducación (información relacionada al proceso salud- enfermedad ajustada a las necesidades del paciente y que promueven un cambio en el comportamiento), estrategias de distracción cognitiva (focalización de la atención voluntaria en pensamientos o imágenes incompatibles a los pensamientos disfuncionales) o 
instrumental (focalización de la atención voluntaria en conductas alternativas), el entrenamiento en habilidades comunicativas y asertividad (moldear y modelar elementos verbales y no verbales de interacción para lograr un objetivo sin dañar los derechos de los demás) y la solución de problemas (la identificación de un problema, generación de alternativas y la valoración de ventajas y desventajas de cada alternativa) resultan de utilidad para la disminución del malestar emocional asociado a la enfermedad y promueven la adherencia al tratamiento médico ${ }^{(12)}$.

Parte del trabajo terapéutico consistió en promover el autoconcepto, autoeficacia e imagen corporal de realizarse la desarticulación. Algo que pudo mitigar el efecto de la notificación del diagnóstico y la probable desarticulación fue que Selene funcionaba como cuidadora primaria de su abuela diabética, la cual estaba amputada de Miembro Pélvico Izquierdo, por lo tanto, imaginaba lo que podría ocurrir y los cambios que podría presentar. Dicha experiencia y la psicoeducación otorgada a la paciente sobre su padecimiento ayudó a modificar las creencias sobre los orígenes de la misma (como consecuencia de un traumatismo) hacia otras más funcionales y realistas.

El establecimiento de componentes de relación con la paciente, sin forzarla a hablar de los temas que no deseaba en una primera instancia, así como el acercamiento logrado mediante la realización de actividades agradables, fue de ayuda para abordar las situaciones que le generaban evitación ansiosa. Ibáñez y Baquero ${ }^{(14)}$ y CelyAranda, Duque y Capafons ${ }^{(13)}$ mencionan a este respecto que el juego y las actividades recreativas y agradables contribuyen a crear un ambiente agradable que favorece el optimismo ante la enfermedad y la hospitalización, desvía la atención de la fatiga, contribuye a la superación del estado de apatía y mejora la salud psicológica y física.

El trabajo con los padres también tuvo un efecto positivo en la paciente al modificar sus formas de interacción. Previo a la intervención, la relación de los padres no era del todo cordial, su comunicación no era asertiva y no podían llegar a acuerdos, sin embargo, con el paso de las sesiones y el entrenamiento en comunicación asertiva con la madre, la psicoeducación sobre la influencia de los componentes físicos, psicológicos y sociales en la percepción del dolor en el padre y solución de problemas con ambos, mejoró su comunicación en torno al tratamiento médico. Es importante hacer notar que, en las primeras sesiones, los padres preferían realizar los cuidados de la paciente solos y en las últimas tres sesiones ambos estaban presentes durante el trabajo con Selene, lo que tuvo un impacto sobre su conducta.

Con la intervención también se logró la toma de decisiones informadas y de manera conjunta de tal suerte que la paciente fue trasladada a otra institución médica en el estado de San Luis Potosí. Parte del trabajo del psicólogo en el ámbito hospitalario es fomentar la resolución de dudas y favorecer la toma de decisiones con la finalidad de que tanto los padres como el mismo niño tomen la mejor decisión para ellos, lo cual fue logrado en este caso. El apoyo social y las interacciones familiares funcionales influyen en la forma en la que los niños se enfrentan a la enfermedad, ayuda a su estado emocional, disminuye el distanciamiento emocional, aumenta la cohesión familiar y promueve el establecimiento de canales de comunicación entre el paciente y sus familiares ${ }^{(7,8,13,14)}$.

Como podemos observar, en el presente caso se obtuvieron resultados favorables, sin embargo, se presentaron circunstancias que lo volvieron complejo. La primera dificultad que se dio a lo largo de esta intervención fue la falta de tiempo para el abordaje de la expresión asertiva de emociones y sentimientos, así como, el trabajo 
sobre autoconcepto y solución de problemas debido a que la paciente fue dada de alta al poco tiempo de haber recibido diagnóstico para recibir tratamiento en otra institución. Otra dificultad fue la aplicación de instrumentos psicométricos para la medición de la sintomatología ansiosa y depresiva, debido a las respuestas de evitación que la paciente presentó en las primeras sesiones y posteriormente a la premura con que se tuvo que realizar la intervención.

Es importante que todo psicólogo clínico dedicado a los cuidados paliativos en pacientes pediátricos tome en cuenta la importancia de estas evaluaciones para confirmar el impacto de su intervención. En el presente caso no se cuentan con esos datos, sin embargo, las verbalizaciones nos sugieren un cambio en el malestar psicológico y por tanto un efecto clínico positivo en Selene y en sus familiares. El valor de estas verbalizaciones también sugiere una modificación en los pensamientos relacionados a la enfermedad y por tanto no pierden en ese sentido su valor subjetivo y cualitativo.

El trabajo psicológico dentro de los CPP permite al niño y a sus familiares afrontar de forma adaptativa la enfermedad y el proceso, en ocasiones largo, que les espera a partir del diagnóstico. Es primordial tomar en cuenta que a pesar de las dificultades que se pudieran presentar durante las primeras etapas de la intervención psicológica, el trabajo debe ser realizado con miras a que las intervenciones no siempre cumplen con la temporalidad adecuada, ya sea por factores médicos, familiares, económicos o por las mismas características del niño. Las intervenciones en este aspecto deben ser realizadas no sólo porque lo dicten organizaciones de salud a nivel mundial o nacional, sino porque es parte del deber del psicólogo que labora en contextos hospitalarios el garantizar el bienestar del niño y su familia.

\section{Referencias bibliográficas}

1. Secretaria de Salud. Centro Nacional para la Salud de la Infancia y la Adolescencia. Cáncer infantil en México. [Acceso el 3 de diciembre de 2017]. Disponible en: https:// www.gob.mx/salud/articulos/cancer-infantil-en-mexico.

2. Organización Panamericana de Salud. [Acceso el 3 de diciembre de 2017]. Disponible en: http://www.paho[Acceso el 3 de diciembre de 2017]..org/hq/index.php?option=com_ docman\&task=cat_view\&gid $=9012 \&$ Itemid $=270 \&$ lang=en .

3. Calvo C, Carboné A, Muñoz A. Cuidados paliativos en oncología pediátrica. En: Gómez Sancho, M. Cuidados paliativos en niños. España: Gafos, 2007.p. 17-42.

4. Castañeda C, O'Shea G, Gallardo D, Farreras D, Calderón M, Chávez E. Cuidados Paliativos: la experiencia en pediatría. Gaceta mexicana de oncología. 2015; 14; 181-4. doi: 10.1016/j.gamo.2015.07.006

5. Salas M, Gabaldón O, Mayoral J, González E, Amayra I. Los cuidados paliativos: un modelo de atención integral al niño gravemente enfermo y a su familia. An Pediatr (Barc). 2004; 61; 330-335.

6. Okhuysen-Cawley, R. Garduño Espinosa A, Paez Aguirre S, Nakashima Paniagua $\mathrm{Y}$, Cardenas-Turanzas M, Reyes Lucas MC et al. Pediatric palliative care in Mexico. En: Knapp Cm Madden V, Fowler-Kerry S, editors. Pediatric palliative care: Global perspectives, Springer Science + Business Media B.V. 2012. p.345-57. doi 10.1007/97894-007-2570-6_19 
7. Quesada L, Masis D. Cuidados paliativos pediátricos: de curar a cuidar. En: Gómez Sancho M, editor. Cuidados paliativos en niños. Lasa Palmas de Gran Canarias: Gafos, 2007.p. 3-16.

8. Mancilla S. Participación de la Psicología en los cuidados paliativos pediátricos. (Tesis de licenciatura). México: Universidad Macional Autónoma de México. 2007.

9. Villegas M. Prevalencia de ansiedad en el paciente con patología oncológica hospitalizado en el servicio de oncología pediátrica del hospital general CMN La Raza. (Tesis de especialidad). México: Universidad Nacional Autónoma de México. 2014.

10. Calvo C, Carboné A, Muñoz A. Cuidados paliativos en oncología pediátrica. En: Gómez Sancho M, editor. Cuidados Paliativos en Niños. España: Gafos, 2007.p. 17-42.

11. Bravo C. Generalidades psicológicas de los padecimientos crónico degenerativos. En Reynoso L, Becerra AL (Comps). Medicina conductual. Teoría y práctica. México: Ed. Qartuppi. 2014.p.43-64.

12. Becerra AL. Manejo de síntomas de ansiedad y depresión en pacientes hospitalizados con cáncer torácico (Tesis de Posgrado no Publicada). Universidad Nacional Autónoma de México. México D.F., 2013.

13. Cely-Aranda J, Duque C, Capafons A. Psicooncología pediátrica. Diversitas: perspectivas en Psicología. 2013; 9: 289-304. doi: 10.15332/s1794-9998.2013.0002.04

14. Ibáñez E, Baquero A. Beneficio del apoyo psicosocial a la calidad de vida de niños y niñas enfermos de cáncer: una revisión sistemática cualitativa. Rev Colomb Enferm 2009; 4: 125-47.

15. Nezu A, Nezu C, Lombardo E. Formulación de casos y diseño de tratamientos CognitivoConductuales. Un enfoque basado en problemas. México: Manual Moderno. 2004. 